\title{
Using an acoustic levitator to investigate the drying kinetics and solids forming process of individual droplets during spray drying
}

\author{
RAMONA HUELSMANN $^{1 *}$ ๑), GUENTER J. ESPER ${ }^{1}$ and REINHARD KOHLUS ${ }^{2}$ \\ ${ }^{1}$ Ramona Huelsmann, University of Applied Sciences Fulda, Germany \\ ${ }^{2}$ University of Hohenheim, Hohenheim, Germany
}

ORIGINAL RESEARCH PAPER

Received: August 19, 2020 • Accepted: October 11, 2020

Published online: November 03, 2020

(C) 2020 The Author(s)

\begin{abstract}
Spray drying is a widely used process to turn slurries into dry powders and is especially important for thermally-sensitive materials, that are often found in the food or pharmaceutical industry. However, detailed insight into the drying kinetics during spray drying is difficult to investigate due to the boundary conditions in a spray drying tower. As a result, there is a lack of important information on the drying process and subsequent solidification of individual droplets. In this context, an experimental setup for a droplet positioned in a stationary ultrasonic field of an acoustic levitator is designed to enable a non-contacting measurement of the drying kinetics and the subsequent solidification process. To generate a comparable situation like in a real spray drying process, the droplet is positioned in an airflow, where air temperature, humidity, and velocity can be adjusted over wide range. Using an infrared camera to measure the surface temperature and a Complementary Metal Oxide Semiconductor (CMOS) camera for object recognition, the droplet can be observed continuously and drying kinetics of the droplet can be determined from the measured surface temperature and decreasing droplet size. Result of a $10 \mathrm{wt} . \%$ aqueous micro particle $\mathrm{TiO}_{2}$ suspension are reported and show that the investigated method is a very valuable and fast tool to safely scale-up spray drying systems very close to real process conditions. Especially when only small sample amounts are available in an early development stage.
\end{abstract}

\section{KEYWORDS}

acoustic levitator, drying kinetics

*Corresponding author. E-mail: Ramona.Huelsmann@lt.hs-fulda.de 


\section{INTRODUCTION}

In food industry spray drying processes are widely used to produce dry free flowing powder starting from a liquid formulation. Since this is a one stage process with high drying capacity it has advantages compared to multi stage processes.

In order to generate large surface areas for fast drying, the slurry is disintegrated in very small droplets which are sprayed into a hot gas stream using nozzles. During the free falling period of the droplets there is a heat and mass exchange between the droplets and hot drying gas. The drying velocity depends on the air conditions (temperature and rel. humidity) and on the size and drying behavior of the product droplets. The latter is mostly responsibly for the length of the spray tower.

Although the drying kinetics of a product is a crucial parameter in this process, the experimental setup to measure the drying kinetics in an environment which comes close to the drying situation in a real spry dryer, is very difficult to establish. With the setup presented here, these investigations can be carried out on single droplets. In this way, preliminary tests for spray drying can be carried out, which are of considerable importance for the research projects involving thermally sensitive products (Breitenbach et al., 2016).

In the recent years, acoustic levitation of single droplets has been developed. So show Eberhardt and Neidhart (1999) the development of an ultrasonic levitator apparatus for sample pretreatment in microanalysis and trace analysis. Also, in the publication from Yarin et al. (1998), Kastner et al. (2001), Schiffter and Lee (2007) or Zaitone (2009) the Levitator assembly is described. The focus here is on the influence of the ultrasonic field on the evaporation rate of the levitated droplets. The thermographic observation of levitated droplets is investigated by Tuckermann et al. (2005) and Wulsten and Lee (2008). They conclude that interpreting the evaporation rate of levitated droplets requires measuring the surface temperature.

With none of these setups reported it is possible to measure the drying kinetics of products in conditions similar to that existing in a spray dryer.

In this work a setup is created for measuring the evaporation rate, which guarantees a very accurate measurement of the surface temperature via infrared thermography and the contour of the levitated droplet via a CMOS camera. The flow conditions with the newly created nozzle are similar to those in the spray dryer and work in the temperature range from ambient temperature to $90{ }^{\circ} \mathrm{C}$ is possible.

Within this project, the drying kinetics of different substance systems can be characterized using individual droplets such combining accurate determination of the drying behavior and the small product amount necessary.

\section{MATERIALS AND METHODS}

An experimental setup for a droplet positioned in a stationary ultrasonic field of an acoustic levitator is designed to enable a non-contacting measurement of the drying kinetics and the subsequent solidification process (Fig. 1).

The ultrasonic levitator is comprised of an ultrasonic transducer and reflector. If US-frequency and distance between transducer and reflector are set correctly, a stationary wave is established. The droplet can be positioned in one of the power nodes and is kept in place by the US-field. 


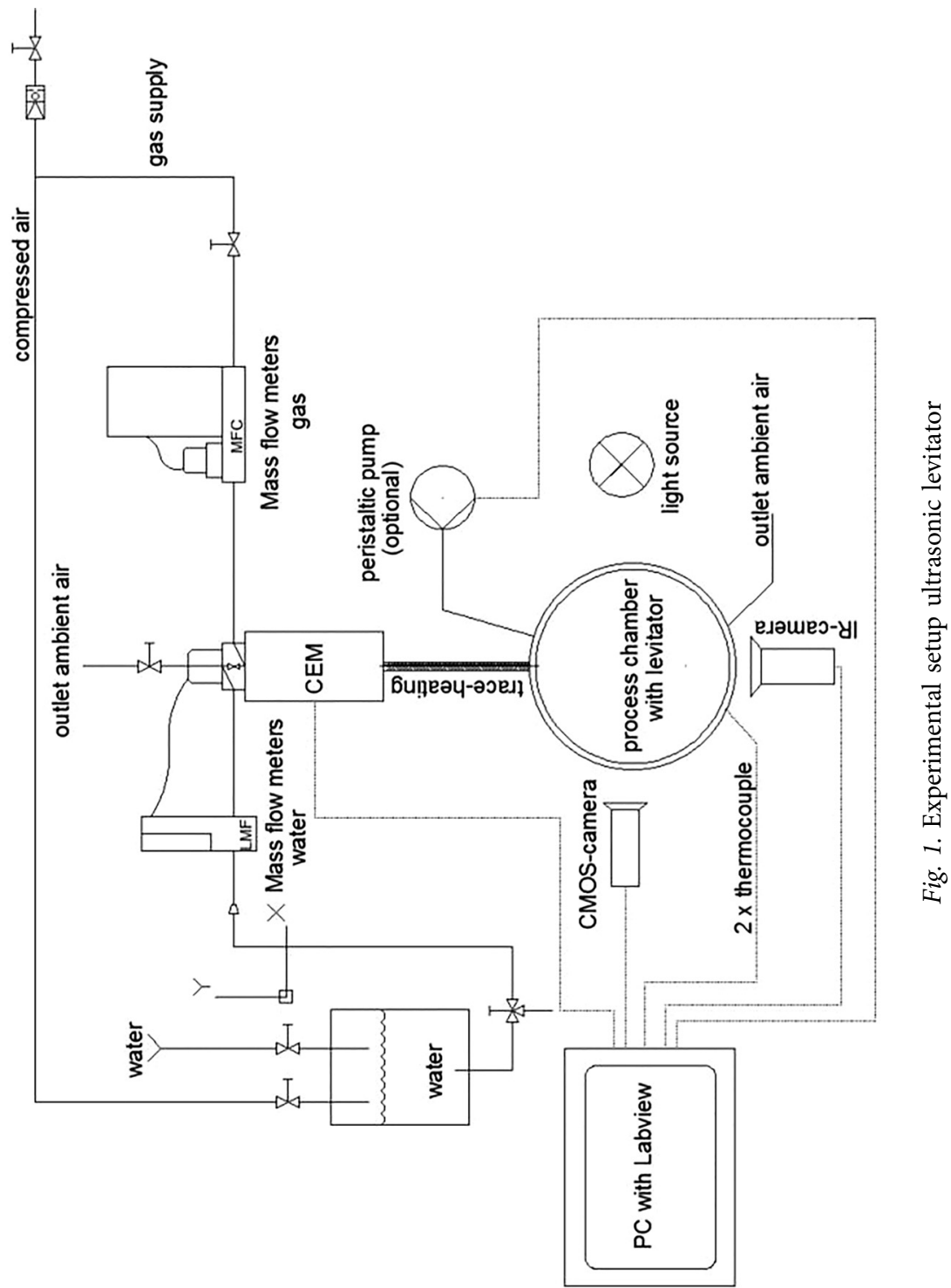


To generate a comparable situation like in a real spray drying process, the reflector has been constructed as a nozzle, which is used to establish an airflow towards the droplet (Fig. 2). The air temperature, humidity and velocity can be adjusted over wide range.

Using an infrared camera to measure the surface temperature and a CMOS-camera for object recognition, the droplet can be observed continuously. The data from the CMOS-camera are used to determine the size of the droplet from which the volume and mass can be derived. The drying velocity in the constant rate period (CRP) can be assumed by the experimentally observed loss of mass in a given time range. During that period (constant rate period: CRP) the surface temperature of the droplet remains at the wet bulb temperature. All the energy that is transferred from the hot gas stream to the droplet is used for evaporation. As soon as the droplet stops shrinking, a rise in surface temperature can be observed and is detected by the infrared sensitive camera (Fig. 3).

During this period (falling rate period: FRP) the heat flow from the gas to the particle stays constant but only a part of the energy is still used for drying. The rest is heating up the droplet.

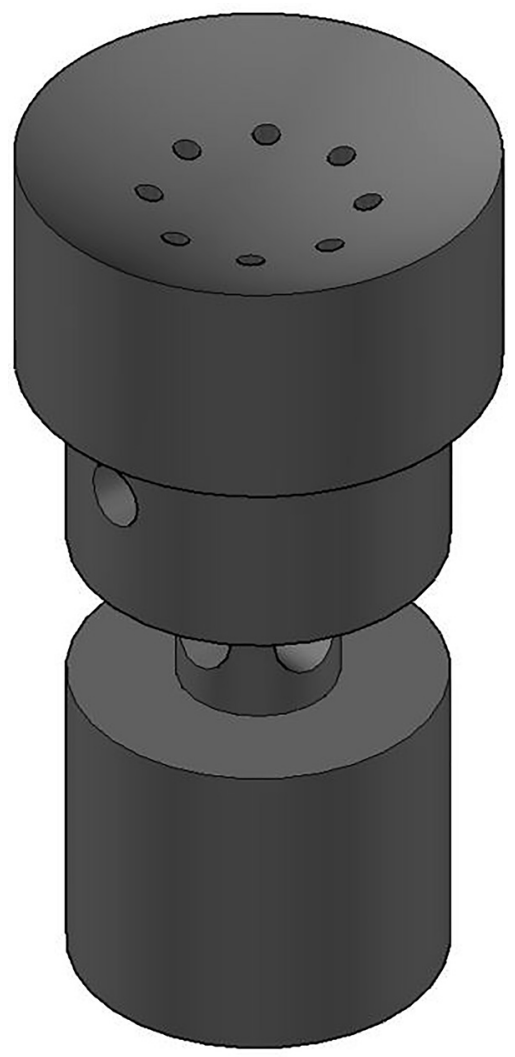

Fig. 2. US reflector modified as nozzle 


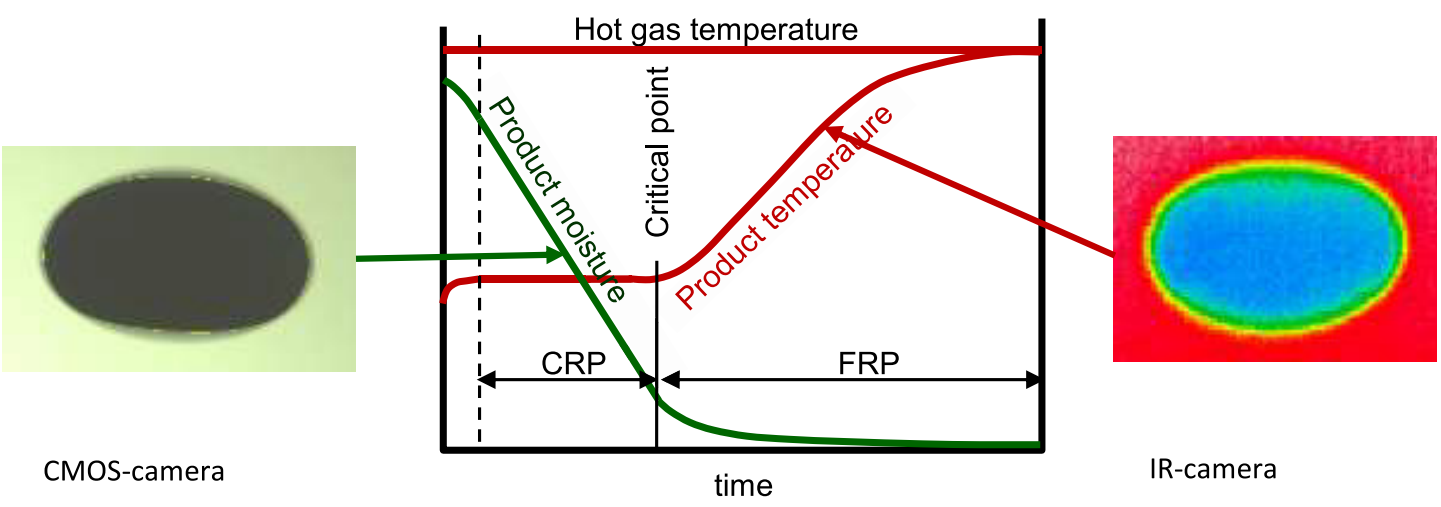

Fig. 3. Determination of the drying kinetics from combined measurement of the volume reduction (moisture content) and the surface temperature of the product 


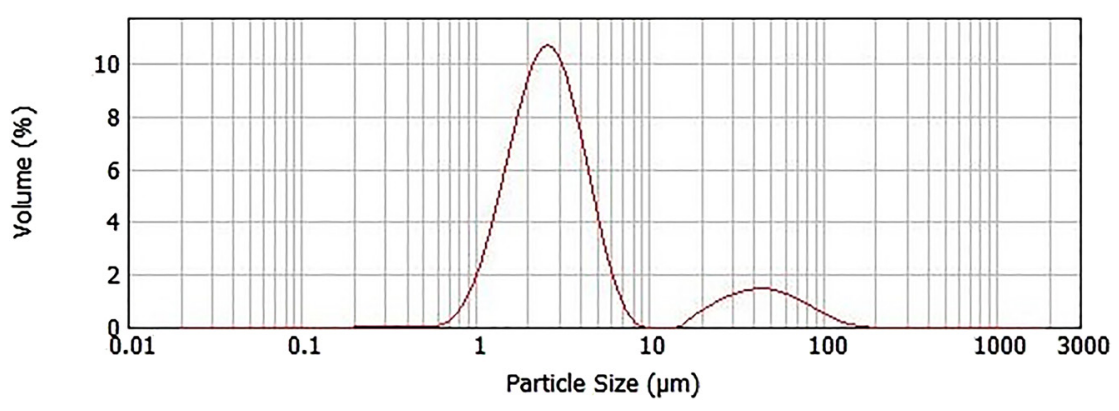

Fig. 4. Particle size distribution $\mathrm{TiO}_{2}$-suspension

From the slope of the temperature rise and the thermodynamic properties of the slurry the drying velocity in the FRP can be calculated.

The drying air is controlled in terms of temperature, relative humidity and velocity. Thus, the complete characteristic drying kinetics of a single droplet in dependence of the major boundary conditions in a spray dryer can be measured.

To start the experiment, the complete setup is heated up to the desired temperature and the droplet is positioned in one of the nodes, using a micro-syringe. After the experiment, the resulting particle is removed from the apparatus for further investigation (final moisture content, strength, etc.)

The product system under investigation is a titanium dioxide $\left(\mathrm{TiO}_{2}\right)$ suspension. $\mathrm{TiO}_{2}$ is supplied by Merck KGaA (CAS-No. 13463-67-7). Fig. 4 shows the primary particle size distribution (PSD). It is obvious that the PSD is a bi-modal distribution, where the bigger particles have an average size of $45 \mu \mathrm{m}$ and the smaller fraction has an average size of $2.5 \mu \mathrm{m}$. The larger particles in the second peak are due to agglomeration.

\section{RESULTS}

$\mathrm{TiO}_{2}$ is suspended into water with an initial water content of $90 \%$. The suspension is positioned in a node of the ultrasonic field. Here it displays a very stable levitation behavior. The resulting $\mathrm{TiO}_{2}$ particle have an almost perfect spherical shape.

Fig. 5 shows a typical result for a drying experiment. Here the surface temperature and the volume of droplet are displayed as a function of time. The volume of the droplet is reducing continuously the drying during the CRP, while the surface temperature stays constant. In the case of the used $\mathrm{TiO}_{2}$ suspension ideal shrinkage can be observed. The critical point where the CRP is switching to the falling rate can easily be detected. Here the shrinkage comes to a stop, because the particle has reached its final size while the temperature starts to rise. Finally, the droplet reaches the surrounding air temperature and the drying experiment is stopped.

The removed particle (Fig. 6) shows a spherical shape. 


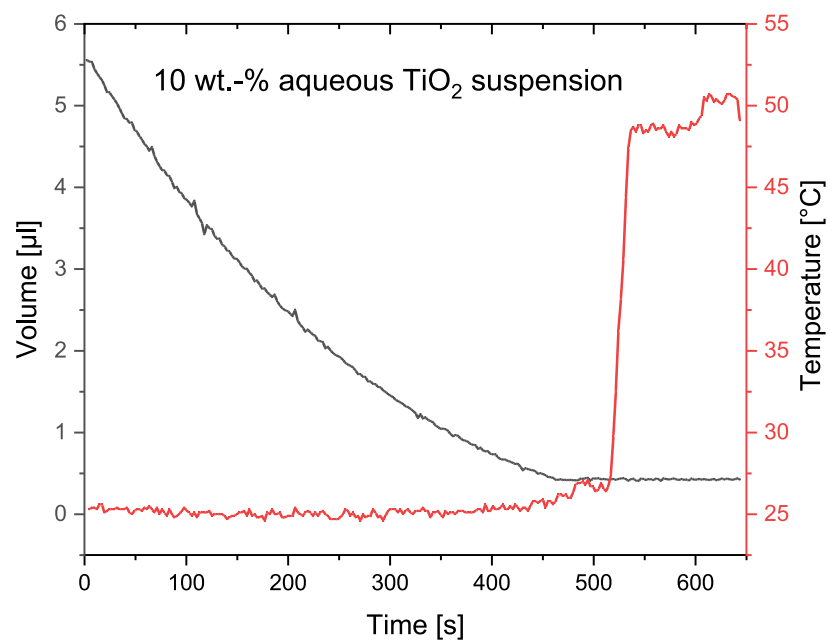

Fig. 5. Results for a $\mathrm{TiO}_{2}$ suspension at an air temperature of $50{ }^{\circ} \mathrm{C}$ and an air velocity of $1 \mathrm{~m} / \mathrm{s}$

\section{DISCUSSION}

Fig. 5 exemplifies that the drying of a 10 wt.\% aqueous $\mathrm{TiO}_{2}$ suspension occurs mainly in the CRP. The loss of size occurs continuously during the drying process and the shrinkage of the droplet stops immediately when the particle solidifies. A time gap of $\sim 130 \mathrm{~s}$ can be observed between the end of the shrinkage and the onset of the temperature rise. This indicates an internal structure with broad capillaries which results in a fast moisture transport to the particles surface. In this case, the particle stays in the CRP regime.

The steep slope of the temperature rise in the FRP regime indicates that no water content is left inside dried particle. Only quite short FRP regimes are detected.

Fig. 6 shows microscopy images (VHX-1000, Keyence, Japan) of obtained dried particles. The surfaces of the resulting particles in Fig. $6 \mathrm{a}$ and $\mathrm{b}$ show different topographies. This result may be traced back to the bi-modality of the PSD and a simultaneous sedimentation process during the drying. Larger particles sediment faster to the bottom of the droplet, resulting in an altered particle surface.

\section{CONCLUSION}

In summary, the implementation of an acoustic levitator for drying kinetics experiments of individual droplets is shown. First experiments prove the applicability of the setup and yield results with high accuracy.

Using a 10 wt.\% aqueous $\mathrm{TiO}_{2}$ micro particle suspension, the different drying phases of the drying process can be measured and identified. Additionally, details of the solidification process during the drying can be observed. Therefore, the acoustic levitator proves to be an effective and 

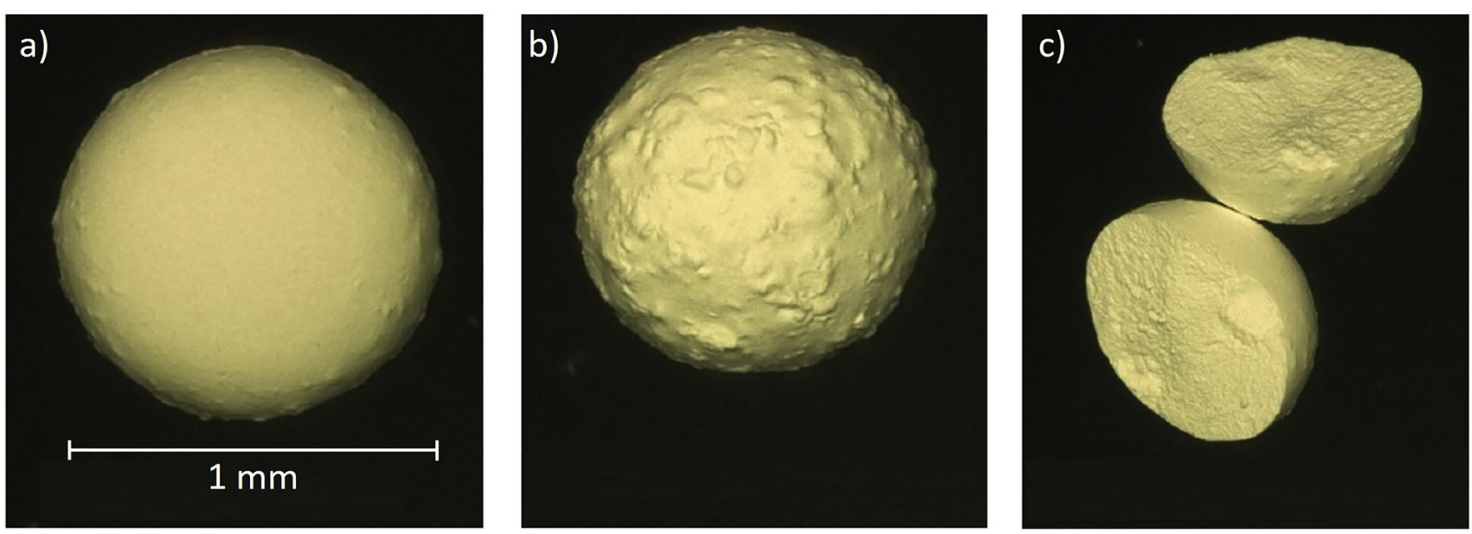

Fig. 6. Dried $\mathrm{TiO}_{2}$ particle (a) top view, (b) bottom view, (c) cross section 
accurate tool to investigate the drying behavior of individual droplets. The investigated method is a very valuable and fast tool to safely scale-up spray drying systems very close to real process conditions, especially when only small sample amounts are available in an early development stage.

\section{REFERENCES}

Breitenbach, J., Beermann, C., and Esper G. J. (2016). Comparison of different methods for microencapsulation of probiotics, Progress in Agricultural Engineering Sciences, 12(1).

Eberhardt, R. and Neidhart, B. (1999) Acoustic levitation device for sample pretreatment in microanalysis and trace analysis. SpringerLink (Online service).

Kastner, O., Brenn, G., Rensink, D., and Tropea, C. (2001). The acoustic tube levitator - A novel device for determining the drying kinetics of single droplets. Chemical Engineering \& Technology, 24(4).

Schiffter, H. and Lee, G. (2007) Single-droplet evaporation kinetics and particle formation in an acoustic levitator. Part 1: evaporation of water microdroplets assessed using boundary-layer and acoustic levitation theories. Journal of pharmaceutical sciences, 96(9).

Tuckermann, R., Bauerecker, S., and Cammenga, H. K. (2005). IR-thermography of evaporating acoustically levitated drops. International Journal of Thermophysics, 26(5).

Wulsten, E. and Lee, G. (2008) Surface temperature of acoustically levitated water microdroplets measured using infra-red thermography. Chemical Engineering Science, 63(22).

Yarin, A. L., Pfaffenlehner, M., and Tropea, C. (1998). On the acoustic levitation of droplets. Journal of Fluid Mechanics, 356.

Zaitone, B. A. A. (2009). Drying of multiphase single droplets in ultrasonic levitator. http://tuprints.ulb.tudarmstadt.de/1892/1/AL_ZAITONE_DISS.pdf.

Open Access. This is an open-access article distributed under the terms of the Creative Commons Attribution-NonCommercial 4.0 International License (https://creativecommons.org/licenses/by-nc/4.0/), which permits unrestricted use, distribution, and reproduction in any medium for non-commercial purposes, provided the original author and source are credited, a link to the CC License is provided, and changes - if any - are indicated. 